\title{
Influence of patterning the TCO layer on the series resistance of thin film HIT solar cells
}

\author{
Romain Champory $^{1}$, Fabien Mandorlo ${ }^{1, a}$, Christian Seassal ${ }^{2}$, and Alain Fave ${ }^{1}$ \\ 1 Université de Lyon, Institut des Nanotechnologies de Lyon INL - UMR 5270 CNRS, INSA Lyon, 69621 Villeurbanne, France \\ 2 École Centrale de Lyon, 36 Avenue Guy de Collongue, 69134 Ecully Cedex, France
}

Received: 25 August 2016 / Received in final form: 23 November 2016 / Accepted: 28 November 2016

(c) R. Champory et al., published by EDP Sciences, 2017

\begin{abstract}
Thin HIT solar cells combine efficient surface passivation and high open circuit voltage leading to high conversion efficiencies. They require a TCO layer in order to ease carriers transfer to the top surface fingers. This Transparent Conductive Oxide layer induces parasitic absorption in the low wavelength range of the solar spectrum that limits the maximum short circuit current. In case of thin film HIT solar cells, the front surface is patterned in order to increase the effective life time of photons in the active material, and the TCO layer is often deposited with a conformal way leading to additional material on the sidewalls of the patterns. In this article, we propose an alternative scheme with a local etching of both the TCO and the front a-Si:H layers in order to reduce the parasitic absorption. We study how the local resistivity of the TCO evolves as a function of the patterns, and demonstrate how the increase of the series resistance can be compensated in order to increase the conversion efficiency.
\end{abstract}

\section{Introduction}

Thin c-Si (mono crystalline silicon) based solar cells are excellent candidates to design light and flexible solar cells requiring a low amount of raw material. Theoretically, their energy conversion efficiencies may reach those of standard cells as long as the thickness of c-Si is at least of the order of $10 \mu \mathrm{m}$ to $20 \mu \mathrm{m}$ [1]. An efficient light trapping structure like a photonic crystal is necessary to enhance the absorption in the active material [1-8]. As surface recombinations are much more critical for thin solar cells, the HIT (Heterojunction with Intrinsic Thin layer) structure is a powerful solution since it currently allows the best conversion efficiency with a c-Si absorber [9]. However, when collectors of minority carriers are located on both sides of the absorber, it is then necessary to add a TCO (Transparent Conductive Oxide) layer to collect carriers into the nearest metal finger. This layer also acts as an anti-reflective coating (ARC), and generally induces parasitic absorption at low wavelengths [5]. Optical simulations demonstrated that the total optical losses in the front a-Si:H and the TCO layers can correspond to a short circuit current density $\left(J_{s c}\right)$ decrease up to $5 \mathrm{~mA} \mathrm{~cm}^{-2}$ for a thin film HIT solar cell [10] or up to $9.3 \%$ for a $1 \mu \mathrm{m}$ thick micro crystalline solar cell [8]. The material used

\footnotetext{
${ }^{a}$ e-mail: fabien.mandorlo@insa-lyon.fr
}

to make the heterojunction itself can lead to additional absorption losses, including when it is made of a-Si:H [5].

Patterning a standard vertical HIT structure by a photonic crystal (PC) is an efficient strategy to strongly increase the photonic current with thin absorbers $[2,3,5,6]$. However, instead of using conformal TCO and collector layers on top of the PC (Fig. 1a), we propose to pattern these two layers. A passivation step is necessary to protect the surface of the photonic crystal (blue element in Fig. 1b). Then, parasitic absorption can be reduced leading to a higher short circuit current [8]. For an ultrathin solar cell (1 $\mu \mathrm{m}$ thick), such an alternative geometry drops down the parasitic absorption from $9.3 \%$ to $2.6 \%$, leading to a $8.1 \%$ increase of the absorption in the active material [8]. However, the contribution of the series resistance corresponding to the travel of carriers towards the metallic fingers in the TCO also increases.

In this article, we investigate the influence of TCO patterning on the series resistance to quantify how much the overall efficiency of new generation solar cells with a front $\mathrm{PC}$ is impacted. First, we will theoretically investigate the influence of TCO patterning on the local series resistance which will be used to calculate its impact on a standard solar cell $\left(156 \times 156 \mathrm{~mm}^{2}\right.$ for instance). We will study how the photonic crystal orientation affects the electro-optical performances of the device. Last, we describe geometries for which the electrical losses could be neglected compared 


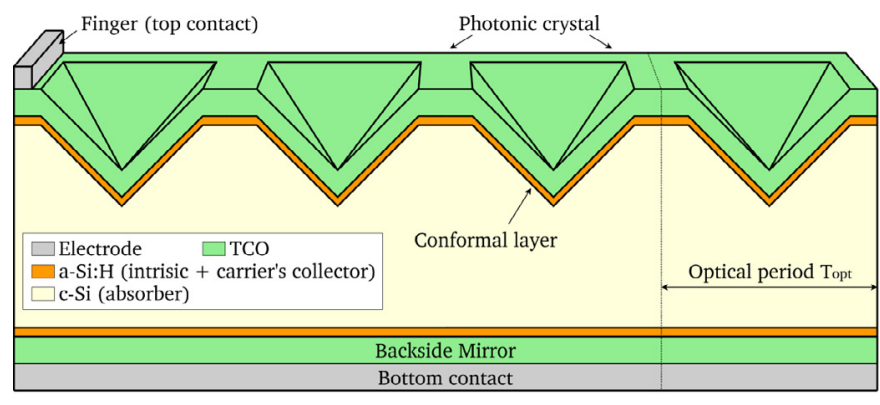

(a) A standard HIT structure

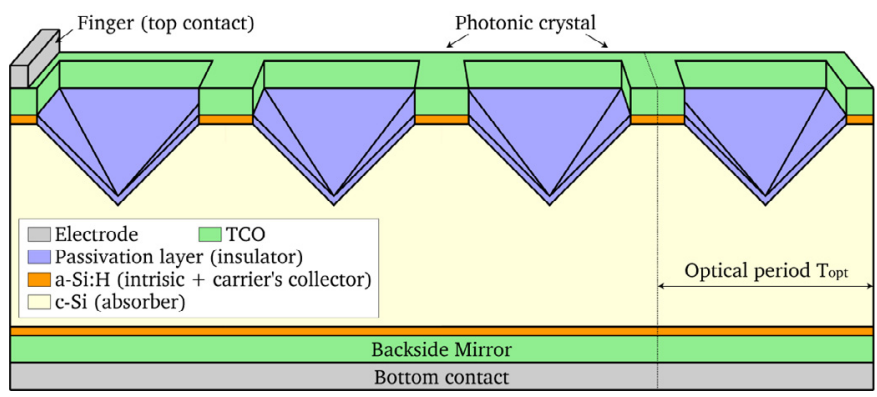

(b) A modified HIT structure with less parasitic absorption

Fig. 1. HIT thin film solar cells with a top photonic crystal (inverted pyramids).

to the absorption increase in the active material, in order to increase the conversion efficiency.

\section{Study of the local sheet resistance}

\subsection{Definition of the electric period}

Figure 2 represents top views of two different solar cells patterned by a square lattice of cylinders holes, with a period $T_{o p t}$. The optical elementary pattern is delimited by dash lines, and its main directions can be aligned (a) or not (b) with the metallic finger. Considering that the cell is infinite in the direction of finger, the minority carriers generated under the PC then join electrodes by the shortest path and encounter a periodic pattern (blue squares in Fig. 2) towards the finger. The period of the electric pattern $T_{e l}$ can be identical with the optical one in case (a) and 45 degrees shifted in case (b) leading to $T_{e l}=\sqrt{2} \cdot T_{\text {opt }}$.

\subsection{Behavior of the local sheet resistance}

The objective is to mimic nanopatterns like inverted nanopyramids (fabricated by wet etching $[1,5,11]$ ) and cylindrical nanoholes (obtained by dry etching $[4,5,11,12])$, covered by a partial TCO layer.

Figure 3 represents the electric periodic elements for a PC made of a square lattice of squares, rounds and diamonds with a top finger aligned (a) or not (b) with the PC directions. All these patterns were simulated using the 2D Silvaco ATLAS module [13].

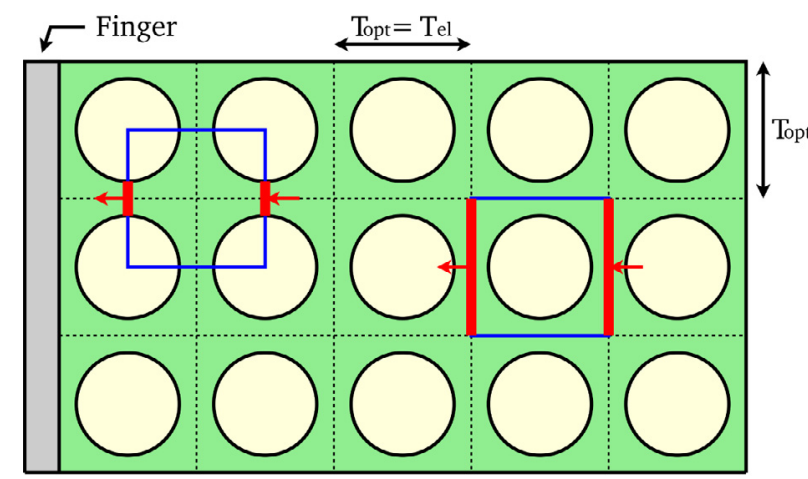

(a) with a top finger aligned on the PC directions

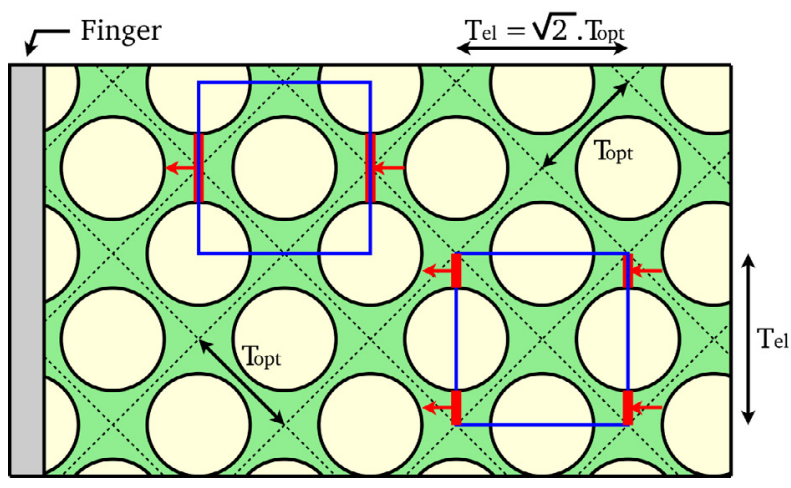

(b) with a top finger at $45^{\circ}$ from the PC directions

Fig. 2. Top view of a square lattice of round holes in the TCO. Blue squares represent the electric periodic pattern and red arrows represent the current flows in direction to the metallic finger.
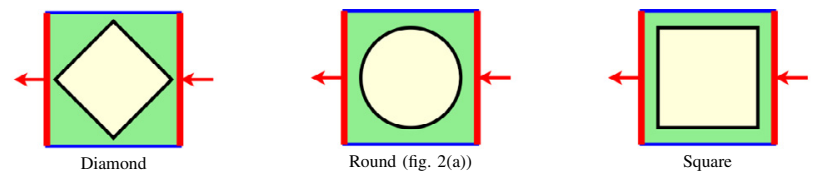

(a) Top finger aligned on the PC directions $\left(T_{e l}=T_{o p t}\right)$
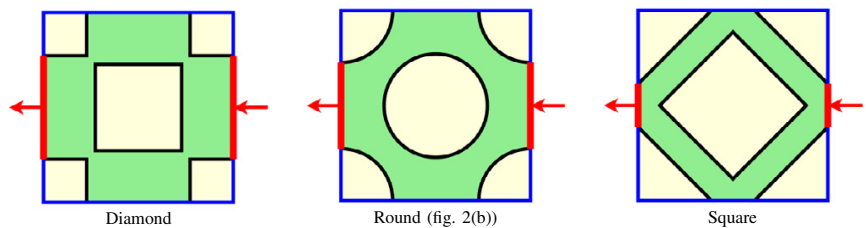

(b) Same PCs with a top finger at $45^{\circ}\left(T_{e l}=\sqrt{2} \cdot T_{o p t}\right)$

Fig. 3. On scale elementary electrical patterns for a square PC.

The conductivity of the TCO layer (including if necessary the top carrier collector) induces an elementary sheet resistance $R_{P C}$ for these lateral current flows. The relative increase of this resistance compared to that one of the unpatterned solution $R_{0}$ will be called the degradation ratio $r=R_{P C} / R_{0}$. By considering a scale factor $k$, both resistances $R_{P C}$ and $R_{0}$ are proportional to $\rho_{0} \cdot k^{-1}$ where $\rho_{0}$ is the resistivity of the patterned material. Consequently, $r$ depends neither on $\rho_{0}$ nor on the size of the square pattern $T_{e l}$. For our study, we assume that the TCO layer 


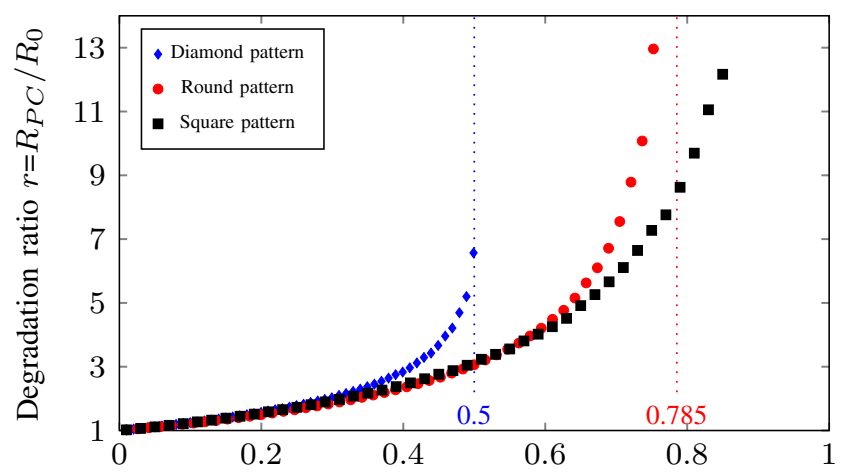

Filling factor $f f$ of the PC (relative amount of etched surface)

Fig. 4. Degradation ratio of the lateral resistance of the TCO layer for different patterns (patterns are simulated with $T_{e l}$ equal to $500 \mathrm{~nm}$ ).

has a constant resistivity even if the transport mechanisms can be more complicated and linked to the presence of traps and tunneling effects at the boundary of its grains in case of ITO $[14,15]$. In this particular material, if the minimum dimension of the elementary sheet resistance is in the range of its grain size the degradation ratio $r$ could be over-estimated since the crystal mobility is higher than that one of the polycrystalline ITO [14]. Since optimal values of $T_{\text {opt }}$ are larger than $500 \mathrm{~nm}$ and $T_{\text {opt }} \leq T_{e l}$, only high values of $f f$ (relative amount of etched TCO Layer) may be impacted, assuming that the grain size is in the same range as the TCO thickness (typ. 60-80 nm) [1, 4, 5, 11, 12].

Figure 4 shows the impact of the filling factor $f f$ on the degradation ratio $r$. These simulations showed that it was not influenced by the orientation of the PC since we had exactly the same results (Fig. 4) for both structures in Figures $3 \mathrm{a}$ and $3 \mathrm{~b}$, even if the electric period $T_{e l}$ is different. Actually, this degradation ratio only depends on the filling factor $f f$ of the pattern.

The results show that for a low $f f$, the impact of the shape is limited, since the degradation is similar in all three cases $(f f<0.3)$. The difference between round patterns or square ones remains limited for $f f$ smaller than 0.5. For PCs requiring a high $f f$ (typ. $>0.6$ ), it is preferable to use a square pattern rather than a round one to minimize $R_{P C}$.

\subsection{Experimental determination of the degradation ratio}

In order to confirm the dependance of $r$ with $f f$, we performed various samples made of a thin patterned $\mathrm{Al}$ layer (fabricated thickness $100 \mathrm{~nm}$ ) on a resistive c-Si substrate, deposited by a lift off process. To ensure compatibility with our LASER lithography setup (Heidelberg $\mu \mathrm{PG} 101)$ in terms of precision, the period $T_{e l}$ is increased up to $50 \mu \mathrm{m}$. Last, thicker metallic pads (200 nm) are locally deposited to collect current (Fig. 5) and we apply the Transmission Line Method (TLM) to measure the patterned layer's resistance. For the sake of technological

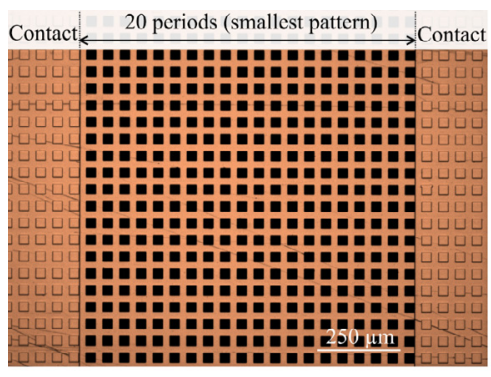

(a) Top view of the two closest metallic pads of one of the TLMs

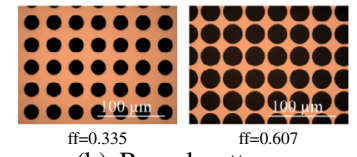

(b) Round patterns

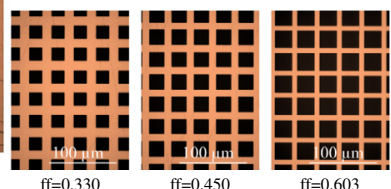

(c) Square patterns
Fig. 5. Microscope views of the fabricated TLMs with different patterns and $T_{e l}$ equal to $50 \mu \mathrm{m}$. Scales for pictures in (b) and (c) are the same.
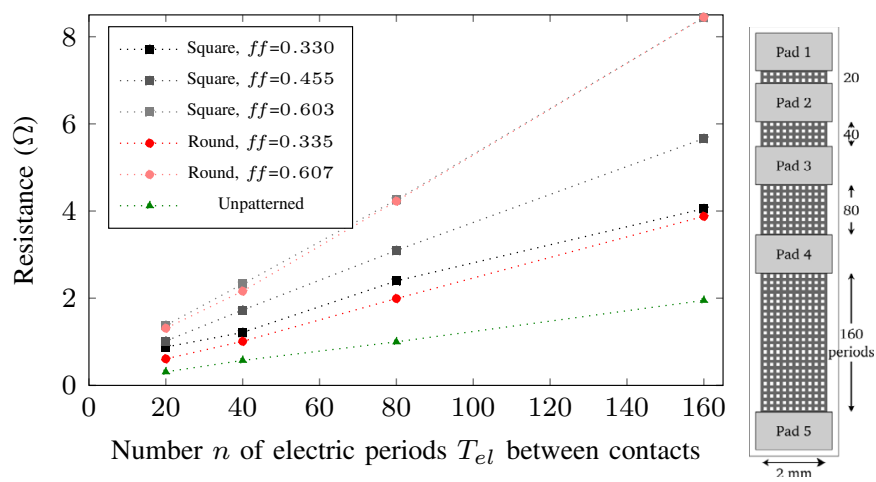

Fig. 6. TLM measurements on patterned Al layers with a pattern aligned with the metallic pads.

Table 1. Degradation ratio obtained experimentally with a $100 \mathrm{~nm}$ thick Al layer. The theoretical values are calculated using the measured filling factor.

\begin{tabular}{|c|c|c|c|c|}
\hline \multirow{2}{*}{$\begin{array}{l}\text { Shape of } \\
\text { the pattern }\end{array}$} & \multicolumn{2}{|c|}{ Filling factor $f f$} & \multicolumn{2}{|c|}{ Degradation ratio $r$} \\
\hline & Measured & Expected & Experiment & Simulation \\
\hline \multirow{3}{*}{ Square } & 0.330 & 0.360 & 1.99 & 2.08 \\
\hline & 0.455 & 0.500 & 2.85 & 2.80 \\
\hline & 0.603 & 0.640 & 4.35 & 4.17 \\
\hline \multirow{2}{*}{ Round } & 0.335 & 0.360 & 2.03 & 2.00 \\
\hline & 0.607 & 0.640 & 4.43 & 4.43 \\
\hline
\end{tabular}

simplicity, we have chosen Aluminum since it is compatible with a lift-off process.

Using a four probe measurement setup to limit the influence of the contact resistivity, we can plot Figure 6 for different filling factors $f f$ and shapes of the holes.

All the TLM devices were made identically, on the same substrate with a controlled number of periods $n$ between each pads, and same width $W$, except for the shape of the elementary pattern and its $f f$. As a consequence, the metal thickness $t_{0}$ is the same for all the characterized devices and $n_{W}=W / T_{e l}$ patterns are present in parallel.

Slopes in Figure 6b can be used to extract the resistance of the elementary pattern since it is equal to $R_{P C} / n_{W}$ for the patterned TLMs and $R_{0} / n_{W}$ for the unpatterned one. Consequently, their ratio corresponds to the measured degradation ratio $r=R_{P C} / R_{0}$ (Tab. 1 ). 


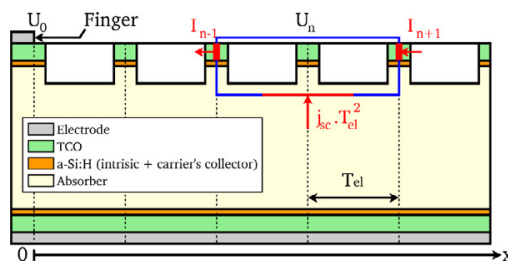

(a) Sectional view

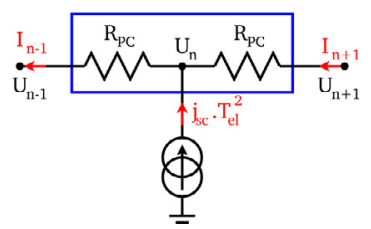

(b) Electric model
Fig. 7. Sectional view of the patterned HIT solar cell.

In order to provide an accurate estimation of $r$, we measured the average size of the patterns with SEM observations and we interpolated datas from Figure 4. Table 1 shows a very good agreement between the expected and experimental degradation ratios $r$ with a maximum difference of $5 \%$.

\section{Impact of the patterns: from the local resistance to the series resistance of a large solar cell}

In this part, we calculate the potential at the TCO surface and then deduce from the distance $p$ between two metallic fingers the front side series resistance of a large solar cell.

\subsection{TCO layer modeling with a resistance array}

Figure 7 represents a HIT solar cell with a top surface photonic crystal. Every elementary electrical pattern produces a small photogenerated current $j_{s c} \cdot T_{e l}^{2}$. In between each pattern, the lateral resistance corresponds to $R_{P C}$, as described in the electrical circuit of Figure 7b.

Around node $n$ of the resistance array, the application of the Kirchhoff's current law states:

$$
\frac{U_{n}-U_{n-1}}{R_{P C}}=\frac{U_{n+1}-U_{n}}{R_{P C}}+j_{s c} \cdot T_{e l}^{2}
$$

At position $N$, the node position is assumed to be located just in between two fingers, so that only half the current produced by an elementary pattern contributes to the ohm law:

$$
U_{N-1}-U_{N}=-\frac{1}{2} \cdot j_{s c} \cdot R_{P C} \cdot T_{e l}^{2} .
$$

At the finger, when the solar cell is short circuited, current $I_{0}$ is equal to the sum of the photogenerated current $j_{s c}$. $T_{e l}^{2} \cdot(N-1 / 2)$, so the ohm law leads to the following initial condition:

$$
U_{0}-U_{1}=-j_{s c} \cdot R_{P C} \cdot T_{e l}^{2} \cdot\left(N-\frac{1}{2}\right)
$$

Then, the solution of equations (1)-(3) with an arbitrary finger potential $U_{0}$ is:

$\forall n \in[0, N], \quad U_{0}-U_{n}=j_{s c} \cdot R_{P C} \cdot T_{e l}^{2} \cdot \frac{N^{2}-(n-N)^{2}}{2}$.

This equation gives the potential at the TCO surface between two fingers when the solar cell is short circuited.

\subsection{Expression of the series resistance}

Thanks to the surface potential $U_{n}$, one can calculate the total dissipated power $P_{j}$ by Joule effect in the entire patterned TCO layer:

$$
P_{J}=\frac{1}{R_{P C}} \cdot \sum_{n=1}^{N}\left(U_{n}-U_{n-1}\right)^{2}
$$

This equation leads to:

$$
P_{J}=\frac{j_{s c}^{2} \cdot T_{e l}^{4} \cdot N^{2} \cdot R_{P C}}{3} \cdot\left(N-\frac{1}{4 \cdot N}\right) .
$$

Let's introduce the series resistance $R_{S}^{1}$ for a single strip of resistances so that it dissipates $P_{j}$ when delivering the total photogenerated current $I_{0}$. This resistance depends on the orientation of the PC since the width of the strip is linked to $T_{e l}$. Then,

$$
P_{J}=R_{S}^{1} \cdot\left(j_{s c} \cdot T_{e l}^{2} \cdot\left(N-\frac{1}{2}\right)\right)^{2}
$$

Then, assuming $N \gg 1$, we get:

$$
R_{S}^{1}=\frac{R_{P C}}{3} \cdot \frac{2 \cdot N^{2}+N}{2 \cdot N-1} \approx N \cdot \frac{R_{P C}}{3} .
$$

One may notice that this elementary resistance $R_{S}$ is different from the sheet resistance (equal to $N \cdot R_{P C}$ ) of the same patterned layer. Indeed, the current sources are regularly spread on the length of the layer, instead of being applied on two opposite cross sections.

If we neglect the influence of the bus bars connecting each fingers, a solar cell with a surface $S$ contains $S /\left(N \cdot T_{e l}^{2}\right)$ elementary stripes in parallel with an elementary series resistance $R_{S}^{1}$. The length of these stripes is also equal to half the pitch $p / 2=N \cdot T_{e l}$. As a result, the series resistance $R_{S}$ of such a solar cell is given by:

$$
R_{S}=\frac{R_{P C} \cdot p^{2}}{12 \cdot S}=\frac{r \cdot R_{0} \cdot p^{2}}{12 \cdot S}
$$

In case the lateral conduction is achieved in a single layer of resistivity $\rho_{0}$ and thickness $t_{0}$ (like a TCO), this last equation is also equal to:

$$
R_{S}=\frac{r \cdot \rho_{0} \cdot p^{2}}{12 \cdot t_{0} \cdot S} \text { with } R_{0}=\frac{\rho_{0}}{t_{0}}
$$

since the sheet resistance $R_{0}$ is also equal to $\rho_{0} / t_{0}$ for a square pattern with width and length equal to $T_{e l}$.

From equations (9) or (10), the additional front series resistance is proportional to both the square of the pitch $p$ and the perturbation of the local series resistance $r$. It is worth to notice that it does not depend on the orientation of the photonic crystal (Fig. 2), even if the electric period $T_{e l}$ may vary. 


\section{Degradation of the conversion efficiency of a large solar cell}

Simulating a solar cell patterned by a PC to study the influence of the pattern on the series resistance is unfortunately not possible, since it would be necessary to simulate thousands of periods of the $\mathrm{PC}$, which would require too much memory and computation time (even for a 2D simulation). However, equation (10) can be exploited to get the $I-V$ curve of a realistic solar cell, and so its conversion efficiency, with the simulation of a single period of the PC and its "local" electric characteristic.

\subsection{Estimation of the $J_{s c}$ gain}

From [11], cylindrical holes lead to a significantly lower $f f$ than reverse pyramids, and so to a lower degradation ratio $r$ (Fig. 4). For this reason, we focus on cylindrical nanoholes optimized by 3D FDTD (Finite Difference Time Domain) simulations. As the parasitic absorption occurs in the short wavelengths, we consider an ultra thin HIT solar cell $(1.1 \mu \mathrm{m})$ to maximize its photogeneration rate in the active layer. In order to get a more realistic estimation of the $J_{s c}$ gain than in [8], we also take into account the a-Si:H layers dispersion characteristics.

When all the top surface is covered by the TCO and a-Si:H layers (Fig. 8a), the thickness of these layers on the sidewalls is proportional to the thickness on the front and bottom surfaces of the pattern with a ratio extracted from experimental observations [5]. When patterning both the TCO and a-Si:H layers (Fig. 8b), the passivation layer is not taken into account since it does not absorb light and its thickness $(\leq 10 \mathrm{~nm}$ ) can be neglected compared to the considered wavelength range. The optimization is applied on the thickness of the top and bottom a-Si:H and TCO layers and on the geometry of the pattern (period, size and depth). The optimal configurations correspond to Figure 8. Whatever the patterning is, the convergence is reached for the same period $(700 \mathrm{~nm})$, but the backside mirror is slightly thicker in case of patterned TCO and aSi:H layers. For both structures, the diameter of the hole in the c-Si layer is quite similar: $490 \mathrm{~nm}$ and $480 \mathrm{~nm}$, respectively. The optimized filling factor is equal to 0.37 , leading to $r \approx 2.15$ from Figure 4 .

The photonic current densities calculated in each layer (Tab. 2) demonstrate that all the photons that are not absorbed in the front TCO and a-Si:H layers are efficiently collected by the active layer. Indeed, the absorption in these two top layers is reduced by $0.3+0.8=1.1 \mathrm{~mA} \mathrm{~cm}^{-2}$ : this value matches the increase of the absorption in the c-Si absorber.

As a consequence, for cylindrical holes, even taking into account the presence of the a-Si:H layers that are more absorbing than the c-Si layer, we can still expect a current density gain higher than $1 \mathrm{~mA} \mathrm{~cm}^{-2}$ thanks to a lower parasitic absorption in the blue range of the solar spectrum.

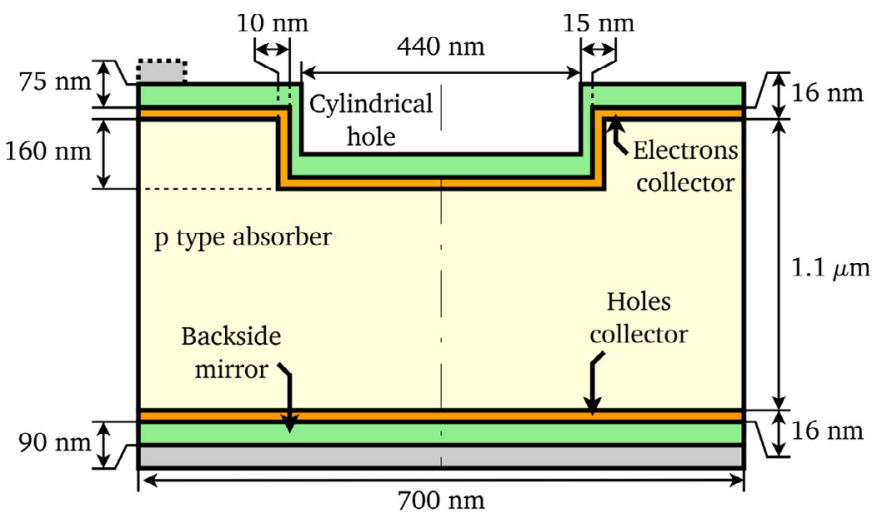

(a) Without TCO (ITO) and a-Si:H patterning

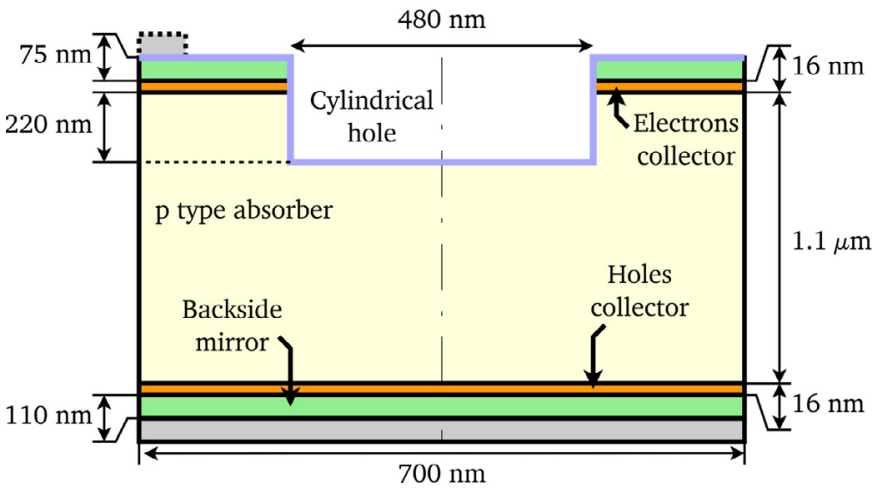

(b) With TCO (ITO) and a-Si:H patterning.

Fig. 8. Description of the two optimized structures by $3 \mathrm{D}$ FDTD and genetic algorithm for a $1.1 \mu \mathrm{m}$ thick HIT solar cell (same legend as Fig. 1).

Table 2. Photonic current densities calculated by integration of the absorbed photons in each layer $\left(\mathrm{mA} \mathrm{cm}^{-2}\right)$.

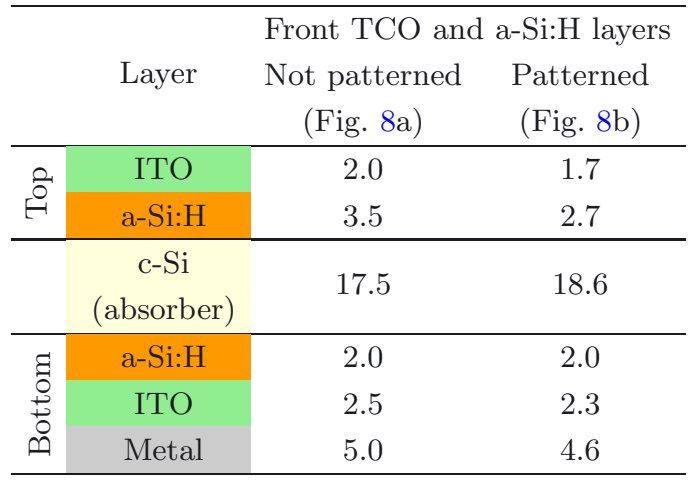

\subsection{Estimation of the conversion efficiency losses in a large solar cell}

In order not to depend on the geometry of the PC, we will consider an ideal solar cell ruled by its open circuit voltage $V_{o c}$, its short circuit current $I_{s c}$ and its ideality factor $n$. As the series resistance $R_{S}$ does not influence both $V_{o c}$ and $I_{s c}$ for any solar cell, the following parts will focus on the conversion efficiency. Then, at first approximation, the current $I$ and the voltage $V$ of such a solar 
Table 3. Parameters of an ideal efficient solar cell with a top TCO layer.

\begin{tabular}{|c|c|c|c|}
\hline & Parameter & Value & Unit \\
\hline \multirow{6}{*}{ 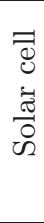 } & Open circuit voltage $V_{o c}$ & 0.750 & $\mathrm{~V}$ \\
\hline & Short circuit current $I_{s c}$ & 4.02 & A \\
\hline & Ideality factor & 1.15 & - \\
\hline & (to match the conversion efficiency) & & \\
\hline & Surface $S$ & 101.8 & $\mathrm{~cm}^{2}$ \\
\hline & Temperature $T$ & 25 & ${ }^{\circ} \mathrm{C}$ \\
\hline 0 & Thickness $t_{0}$ & 75 & $\mathrm{~nm}$ \\
\hline$E$ & Resistivity $\rho_{0}$ & 540 & $\mu \Omega \mathrm{cm}$ \\
\hline
\end{tabular}

cell at temperature $T$ are linked by;

$$
I=I_{s c} \cdot\left(1-\frac{e^{\frac{q \cdot\left(V-R_{S} \cdot I\right)}{n \cdot k_{B} \cdot T}}-1}{e^{\frac{q \cdot V_{o c}}{n \cdot k_{B} \cdot T}}-1}\right)
$$

derived from:

$$
I=I_{s c}-I_{d a r k} \cdot\left(e^{\frac{q \cdot\left(V-R_{S} \cdot I\right)}{n \cdot k_{B} \cdot T}}-1\right)
$$

where the dark current $I_{\text {dark }}$ has been expressed as a function of $V_{o c}$ since it corresponds to $I=0$.

Equation (11) can be used to express the voltage $V$ as a function of the current $I$, and so, the available electric power $P$ :

$P=\frac{n \cdot k_{B} \cdot T \cdot I}{q} \cdot \ln \left(\left(1-\frac{I}{I_{s c}}\right) \cdot e^{\frac{q \cdot V_{o c}}{n \cdot k_{B} \cdot T}}+\frac{I}{I_{s c}}\right)-R_{S} \cdot I^{2}$

Then, the maximum conversion efficiency $\eta$ of this ideal solar cell with a serial resistance $R_{S}$ can be found from this last expression by calculating the maximum of $P(I)$.

In order to maximize the influence of $R_{S}$, we will consider an efficient solar cell with a $V_{o c}, j_{s c}$ close to the actual record for a HIT structure [16], with the same conversion efficiency $\eta_{0}=24.7 \%$. We assume that a $75 \mathrm{~nm}$ thick ITO layer is responsible for the front series resistance increase. Finally, all the parameters are summed up in Table 3.

Figure 9 represents the degradation of the conversion efficiency $\eta$ of this ideal solar cell as a function of the pitch $p$ between the fingers and the degradation ratio $r$ with the same vertical axis as for Figure 4 . The cases where $r=1$ describe the evolution of the conversion efficiency for a TCO covering all the pattern (Fig. 1a). When $r>1$, the solar cell looks like Figure 1b with partial TCO and aSi:H layers. White contour lines obtained thanks to equations (9) show that for a given degradation ratio $r$, the conversion efficiency of the solar cell with a patterned TCO is the same as for the un-patterned one if both the pitch $p$ and the width of the fingers (same optical shading) are divided by $\sqrt{r}$. Consequently, patterning the TCO layer can be compensated by reducing the distance between two

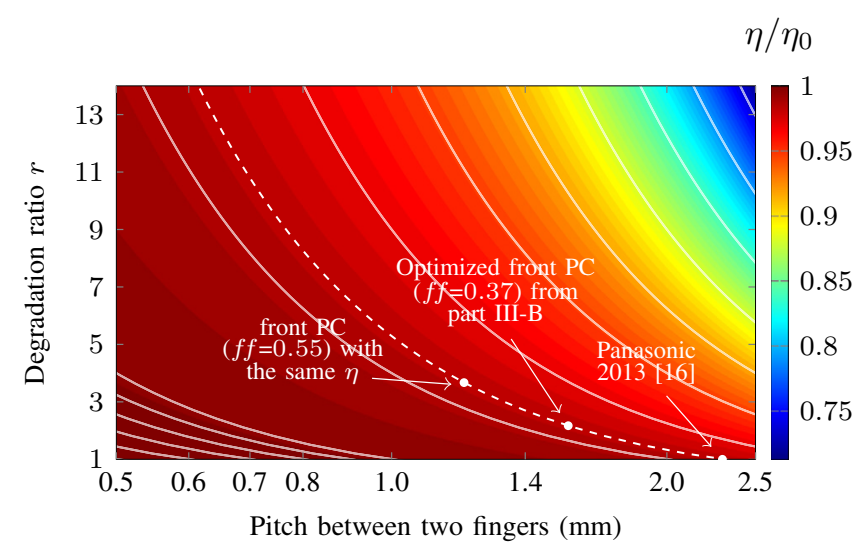

Fig. 9. Relative degradation of the conversion efficiency for an ideal efficient solar cell (see Tab. 3). Only the front series resistance is taken into account to calculate the conversion efficiency degradation. White lines are contour levels.

fingers to prevent the increase of the series resistance. Reducing the fingers width with the same ratio $\sqrt{r}$ would avoid extra shadowing, so parasitic absorption in the top metal layer would stay unchanged. Furthermore, by also scaling their width, Joule losses along the fingers are unchanged, with no additional electrical losses. Then, the solution presented in Figure 1b can be used to reduce the parasitic absorption in the TCO, and, under the condition that a smaller pitch with thinner fingers can be achieved, its conversion efficiency can be higher than for a solar cell like Figure 1a.

For instance, if we consider that a $\mathrm{PC}$ patterned thin film solar cell can reach the same performance as the Panasonic reference (pitch estimated at $2.3 \mathrm{~mm}$ ) [16], then a filling factor of 0.55 (pessimistic value since the previous part shows that it could be as low as 0.37 ) leads to a degradation ratio $r \approx 3.6$ (Fig. 4). Consequently, the minimum pitch is $1.2 \mathrm{~mm}$ for an ITO layer with a resistivity of $540 \mu \Omega \mathrm{cm}$. The width of the fingers should also be divided by a factor $\approx 1.9$ to keep the same shadowing and series resistance contribution of the electrical fingers. According to the International Technology Roadmap for Photovoltaic (ITRPV), it is expected that this $50 \%$ decrease of the fingers' width is reached in 10 years, passing from 45 to $22.5 \mu \mathrm{m}$ [17].

On the other hand, if technological considerations lead to constraints in terms of fingers width and periodicity, one can define a threshold value of the pitch $p_{t h}$ for which the increase of the photogeneration is balanced by the increase of the series resistance in the TCO. This particular point depends on the solar cell itself (more particularly its $\left.J_{s c}\right)$. The main consequence is that our structure can be more efficient if $p<p_{t h}$. For instance, using the extreme example used for Figure 9 with a pitch of $1.5 \mathrm{~mm}$ (instead of $1.2 \mathrm{~mm}$ ) for a $\mathrm{PC}$ with a $f f$ equal to 0.55 induces a relative $2.4 \%$ conversion efficiency loss. In that case, the benefit of a $1 \mathrm{~mA} \mathrm{~cm}^{-2}$ current density increase would be compensated by the increase of the electrical losses, so the pitch should be chosen between 1.2 and $1.5 \mathrm{~mm}$ to increase the solar cell conversion efficiency. However, 
the previous part suggests that the optimal $f f$ of the $\mathrm{PC}$ could be lower than 0.55 , requiring a scale factor $\sqrt{r} \approx 1.4$ : a $32 \%$ reduction of the pitch and finger's width is enough to ensure that no impact on the series resistance is introduced. Consequently, it should be possible to pattern the TCO so that the optical gain corresponds to a minimum $+1 \mathrm{~mA} \mathrm{~cm}^{-2}$ increase of $J_{s c}$. Then, our solution is still more interesting even if the pitch is not as low as possible.

Last, one may notice that for thin film HIT solar cells with a thinner c-Si absorber, the short circuit current is actually limited to lower values that reduce the impact of the series resistance, leading to less constraint on the pitch and the width of the fingers.

\subsection{Influence of the pattern' shape}

Reference [11] demonstrates that for reverse pyramids, the filling factor $f f$ must be around 0.9 to maximize the photon current density in the active material. From Figure 4 and Table A.1, this condition typically corresponds to $r \approx 19$. On the contrary, with a pattern of cylinder holes, the optimal $f f$ is in the range $0.3-0.6$, leading to smaller degradation ratio (typ. $r<4.3$ ). Using Figure 9, we can deduce that for cylinder holes, the ideal pitch and width of the fingers is closer to the industrial standard processes.

The contour lines of Figure 9 and the relation between filling factor and the degradation ratio (Fig. 4) can be used as an abacus to directly obtain the pitch of the patterned TCO solar cell with the same series resistance. In that case, using equations (9) or (10) and a the simulation of a single period of the photonic crystal, the additional series resistance to be taken into account to convert a local $I-V$ curve into that one of a large device can be calculated. Then, the conversion efficiency of the large solar cell can be calculated.

\section{Conclusion}

We report on the increase of the series resistance in thin HIT solar cells when patterning the front TCO layer. We demonstrate that this degradation of the electric properties only depends on the shape and filling factor of the pattern, and not its orientation towards the position of the electric fingers.

We also explain that a single local simulation can be used to calculate the series resistance of a large solar cell, and its conversion efficiency, even taking into account the patterning of the TCO layer.

Last, we demonstrate that the increase of the series resistance can be compensated by lowering the distance between fingers and their width. Then, for a HIT solar cell with a periodic photonic crystal, the increase of the absorption in the active material thanks to the reduction of the parasitic absorption can be completely used to increase the conversion efficiency. The only limitation is the capacity to reduce the finger's width to prevent the increase of optical shadowing. Consequently, the lower parasitic absorption in the TCO layer of a thin HIT solar cell
Table A.1. Coefficient to approximate $r^{-1}(f f)$ with a maximum relative difference of $5 \%$ compared to the simulations (Fig. 4).

\begin{tabular}{cccc}
\hline Pattern & Diamond & Round & Square \\
\hline$f f$ range & {$[0,0.489]$} & {$[0,0.754]$} & {$[0,0.949]$} \\
$r$ range & {$[1,4.977]$} & {$[1,12.62]$} & {$[1,42.27]$} \\
\hline$a_{3}$ & 3.2866 & -1.0560 & -0.6656 \\
$a_{2}$ & 2.9322 & 1.8109 & 1.7028 \\
$a_{1}$ & -2.2921 & -1.9870 & -2.0389 \\
$a_{0}$ & 1.0049 & 1.0006 & 0.9938 \\
\hline
\end{tabular}

with a patterned TCO layer can increase the conversion efficiency at the cost of a different front contact design. A gain on the short circuit current density up to $1 \mathrm{~mA} \mathrm{~cm}^{-2}$ is expected.

This work was supported by the project FP7-PhotonVoltaics (Grant No. 309127). The authors would like to thank the Nanolyon technological platform at INL where samples were processed and Nicolas Terrier and Céline Chevalier for their help on the Laser lithography technique.

\section{Appendix}

In order to ease the estimation of the overall performance of a large solar cell from a local simulation, we propose to interpolate $r^{-1}$ with a polynomial expression of the filling factor $f f$ :

$$
r^{-1}(f f)=\sum_{i=0}^{3} a_{i} \cdot f f^{i} .
$$

Then, using the coefficients in Table A.1, one may easily assess the degradation of diamond, round and square patterns in the TCO layer (Fig. 4).

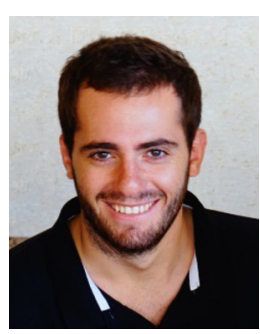

Romain CHAMPORY was born in 1991. He received his engineer diploma from Institut $\mathrm{Na}-$ tional Polytechnique de Grenoble - PHELMA (PHysics, ELectronics, MAterials), Grenoble, France in 2013. After an internship at CEAINES, he joined at the end of 2013 the photovotaic team at Lyon Institute of Nanotechnologies (INL) to start his Ph.D. on thin-film monocrystalline silicon solar cells.

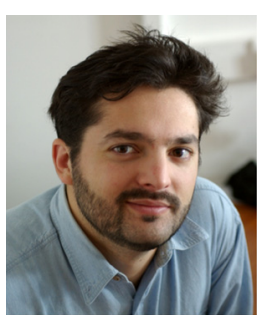

Fabien MANDORLO was born in 1982 . He received his engineer diploma from the École Centrale de Lyon, Ecully, France in 2005, and the same year his M.S. degree in electronics from the University of Lyon. Then, he conducted researches both at CEA-LETI and INL (Lyon Institute of Nanotechnologies). In 2010, he joined the Silicon Photonic team at the Institut d'Electronique Fondamentale (IEF) to work on Si-Ge APD photodiodes for telecom applications. Since 2011, he is part of the photovotaic team of the INL. 


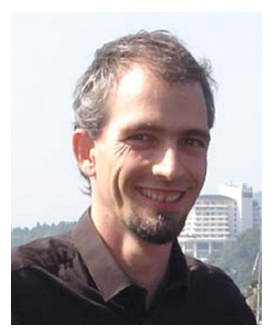

Christian SEASSAL is Senior Researcher at CNRS. He graduated from INSA de Lyon (1993) and received his Ph.D. from École Centrale de Lyon in 1997. His research activities concern photonic nanostructures and their applications for integrated photonics and solar photovoltaics, and he is Deputy Director of the Lyon Institute of Nanotechnology (INL). He has authored and co-authored about 110 research papers in international journals, and of over 50 invited conferences. He is member of the Optical Society (OSA), the Institute of Electrical and Electronics Engineers (IEEE) and the International Society for Photonics and Optics (SPIE). He is deputy editor of the OSA Optics Express Journal. He received the French CNRS bronze medal in 2002.

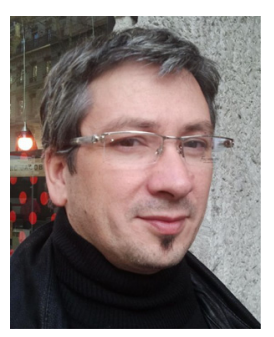

Alain FAVE is Associate Profesor at INSALyon. He graduated at INSA-Lyon as Material Sciences Engineer. He received his Ph.D. in 1996 from INSA-Lyon about III-V Solar cells. After a research fellowship on GaN MBE growth at ETL (Tsukuba, Japan), he joined the Photovoltaic group of INL. His research activities mainly concern Silicon epitaxy and related technogies for thin film solar cells. More recently, he is involved in projects concerning III-V/Si tandem solar cells.

\section{References}

1. A. Mavrokefalos, S.E. Han, S. Yerci, M.S. Branham, G. Chen, Nano Lett. 12, 2792 (2012)

2. J.M. Gee, in Photovoltaic Specialists Conference, 2002. Conference Record of the Twenty-Ninth IEEE (IEEE, 2002), pp. 150-153

3. P. Bermel, C. Luo, L. Zeng, L.C. Kimerling, J.D. Joannopoulos, Opt. Exp. 15, 16986 (2007)

4. X. Meng, V. Depauw, G. Gomard, O. El Daif, C. Trompoukis, E. Drouard, C. Jamois, A. Fave, F. Dross, I. Gordon et al., Opt. Exp. 20, A465 (2012)

5. C. Trompoukis, I. Abdo, R. Cariou, I. Cosme, W. Chen, O. Deparis, A. Dmitriev, E. Drouard, M. Foldyna, E.G. Caurel et al., Physica Status Solidi A 212, 140 (2015)
6. M.S. Branham, W.C. Hsu, S. Yerci, J. Loomis, S.V. Boriskina, B.R. Hoard, S.E. Han, G. Chen, Adv. Mater. 27, 2182 (2015)

7. K. Ishizaki, M. De Zoysa, Y. Tanaka, T. Umeda, Y. Kawamoto, S. Noda, Opt. Exp. 23, A1040 (2015)

8. N.A. Yahaya, N. Yamada, Y. Kotaki, T. Nakayama, Opt. Exp. 21, 5924 (2013)

9. K. Masuko, M. Shigematsu, T. Hashiguchi, D. Fujishima, M. Kai, N. Yoshimura, T. Yamaguchi, Y. Ichihashi, T. Mishima, N. Matsubara et al., Photovoltaics IEEE J. 4, 1433 (2014)

10. R. Champory, F. Mandorlo, L. Lalouat, P. Harouri, A. Roca I Cabarrocas, R. Orobtchouk, A. Fave, E. Fourmond, C. Seassal, Reduction of parasitic absorption in ITO for ultra-thin film c-Si solar cell, in 6th International Conference on Crystalline Silicon Photovoltaics (Silicon PV, 2016)

11. V. Depauw, C. Trompoukis, O. El Daif, L. Lalouat, H. Ding, J. Liu, A. Harouri, R. Orobtchouk, F. Mandorlo, A. Fave et al., When nanophotonics meet thin crystallinesilicon photovoltaics, in 31st European Photovoltaic Solar Energy Conference-EUPVSEC (2015)

12. V. Depauw, X. Meng, O. El Daif, G. Gomard, L. Lalouat, E. Drouard, C. Trompoukis, A. Fave, C. Seassal, I. Gordon, Photovoltaics IEEE J. 4, 215 (2014)

13. ATLAS User's Manual, Device Simulation Software, SILVACO International, Santa Clara (2015)

14. K. Ellmer, R. Mientus, Thin solid films 516, 4620 (2008)

15. K. Ellmer, R. Mientus, Thin Solid Films 516, 5829 (2008)

16. M. Taguchi, A. Yano, S. Tohoda, K. Matsuyama, Y. Nakamura, T. Nishiwaki, K. Fujita, E. Maruyama, Photovoltaics IEEE J. 4, 96 (2014)

17. International technology Roadmap for Photovoltaic (ITRPV) 2015 Results v.2, SEMI Europe, Berlin, Germany (2016), p. 26

Cite this article as: Romain Champory, Fabien Mandorlo, Christian Seassal, Alain Fave, Influence of patterning the TCO layer on the series resistance of thin film HIT solar cells, EPJ Photovoltaics 8, 80101 (2017). 